Musées, Patrimoine et Culture scientifiques et techniques

$127 \mid 2010$

janvier - février 2010

\title{
Le musée d'entreprise horlogère, instrument de communication des marques
}

François Courvoisier et Fabienne Courvoisier

\section{OpenEdition \\ Journals}

Édition électronique

URL : http://journals.openedition.org/ocim/190

DOI : $10.4000 /$ ocim. 190

ISSN : 2108-646X

Éditeur

OCIM

Édition imprimée

Date de publication : 1 janvier 2010

Pagination : 14-24

ISSN : 0994-1908

Référence électronique

François Courvoisier et Fabienne Courvoisier, «Le musée d'entreprise horlogère, instrument de communication des marques ", La Lettre de I'OCIM [En ligne], 127 | 2010, mis en ligne le 01 janvier 2012, consulté le 30 avril 2019. URL : http://journals.openedition.org/ocim/190 ; DOI : 10.4000/ ocim. 190 


\section{Le musée d'entreprise horlogère, instrument de communication des marques}

\section{François Courvoisier et Fabienne Courvoisier *}

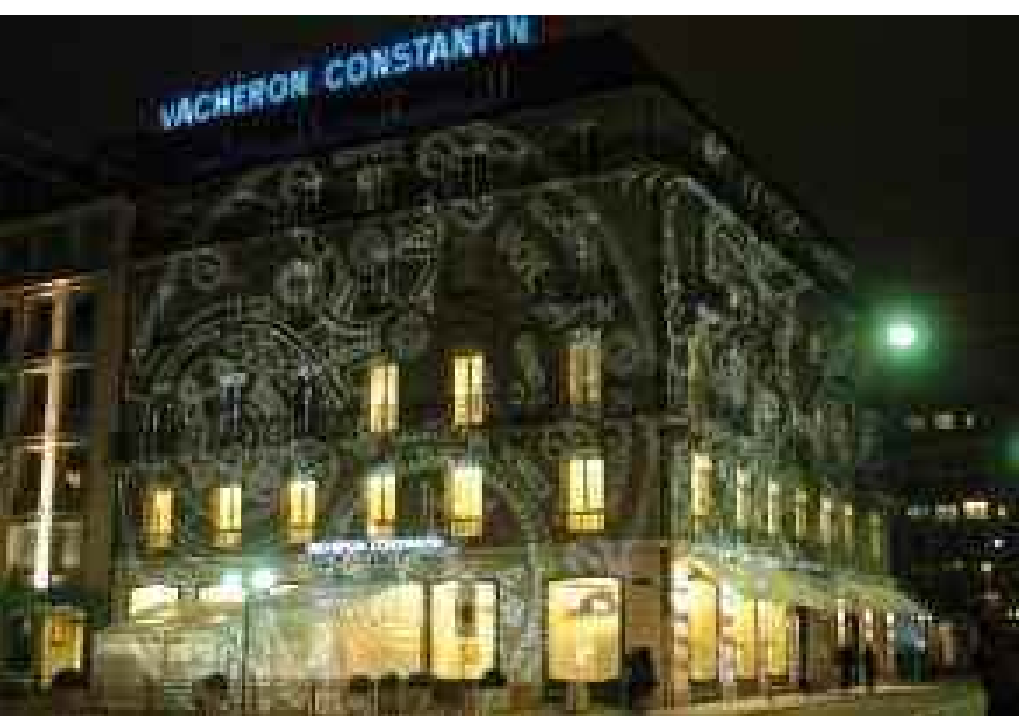

La Maison Vacheron Constantin, à Genève, ne présente qu'une partie de la collection historique de la marque, car celle-ci tourne généralement dans les filiales de la manufacture. (C) Vacheron Constantin

* François Courvoisier est docteur en Sciences économiques, professeur HES à la Haute École de

Gestion Arc de Neuchâtel, chercheur associé de la chaire ACME à l'École de Management de Bordeaux francois.courvoisier@he-arc.ch

Fabienne Courvoisier est licenciée en Sciences économiques, adjointe scientifique à la Haute École de Gestion Arc de Neuchâtel
À partir d'études générales sur les musées $d^{\prime}$ 'entreprise et d'entretiens menés auprès des conservateurs de musées horlogers, les auteurs mettent en évidence que l'ouverture de musées d'entreprises horlogères suisses a un triple but : la conservation et la mise en valeur de leur patrimoine, la légitimation de leur nom dans I'histoire et la promotion de leur marque et de leur région.

En Suisse, en 2008, le secteur de l'horlogerie a exporté des montres pour une valeur ex-usine de 17 milliards de francs (soit environ 11 milliards d'euros), ce qui en fait le troisième secteur d'exportation de Suisse après les machines-outils et la chimie. Pour 2009, suite à la crise financière et économique mondiale, les ventes devraient être en régression d'environ $10 \%$ par rapport à 2008 (Fédération de l'industrie horlogère suisse, FHS, 2009).

\section{Objectif, intérêt et méthodologie de la recherche}

On observe depuis quelques années une augmentation du nombre d'entreprises horlogères qui ont ouvert, entre Genève et Schaffhouse, une galerie ou un musée d'entreprise. Aucune statistique n'existe à ce propos, car ces musées ne couvrent pas toujours les quatre missions définies par l'ICOM (International Council of Museums) : conservation, recherche, formation et divertissement ${ }^{(1)}$. La plupart du temps, ces musées d'entreprise ne font pas de recherche et ils sont rarement ouverts au grand public. De plus, ce ne sont pas des organisations à but non lucratif, sauf si 
l'entreprise les a constitués juridiquement en fondation ou en association. Pour les recenser, nous avons pratiqué une « veille médias » sur la base des quelques 620 entreprises horlogères membres de la FHS. Autour du concept de musée d'entreprise, appelé aussi musée de société ou corporate museum, nous avons constaté que les travaux académiques ne sont pas nombreux, ce qui offre un champ de recherche très intéressant à explorer. Spécifiquement, sur les musées d'entreprises horlogères, il n'y a à notre connaissance pas encore eu de publications académiques.

Une tendance assez nette se dégage quant à la création de nouveaux musées d'entreprise et au développement de collections existantes pour les présenter à divers publics. C'est sur les raisons de ce phénomène que porte notre recherche exploratoire. Notre question de recherche consiste à savoir si les entreprises ouvrent des musées ou galeries simplement pour mieux conserver et présenter leur patrimoine que dans des archives traditionnelles, ou si elles les utilisent activement dans leur stratégie de communication marketing pour promouvoir leurs marques.

Notre recherche se base sur une double méthodologie : recherche bibliographique et entretiens sur le terrain avec des responsables muséaux. Nous avons tout d'abord recherché des articles dans des publications scientifiques traitant de musées d'entreprise, en Suisse, en France et dans les pays anglo-saxons. Nous avons ensuite affiné notre recherche bibliographique en la concentrant plus spécifiquement sur les musées d'entreprises horlogères, puis nous nous sommes approchés de conservateurs de musées d'entreprises horlogères, publics et privés, et les avons interrogés, au moyen d'un guide d'entretien semi directif, sur leurs motivations et leurs visions. Notre échantillon de spécialistes rencontrés peut être qualifié de convenable, avec un souci de diversité : représentants des grands groupes (Swatch, Richemont, LVMH), ainsi que de manufactures indépendantes (Girard-Perregaux, Audemars Piguet, Chopard).

\section{Les musées d'entreprise}

Certains musées d'entreprise ne sont que des « simulacres de musées » visant à des buts surtout mercantiles. La plupart des musées d'entreprise valent heureusement mieux : lieux d'accueil pour le visiteur de passage, ils permettent de connaître les origines de l'établissement, l'histoire des fabrications et des produits, ainsi que les mutations socio-économiques de l'entreprise (Rasse, 1995). On peut définir le musée

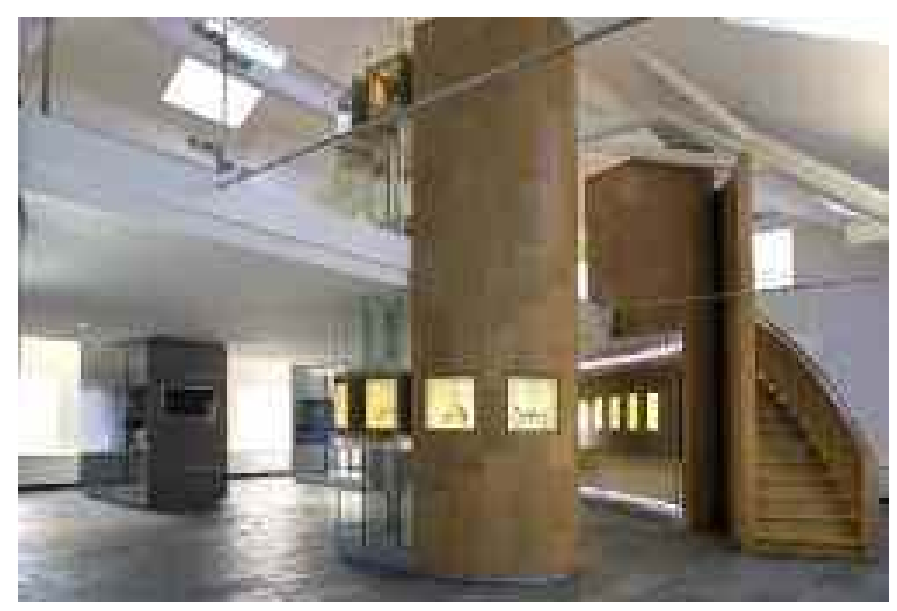

Dans la maison du fondateur Antoine LeCoultre, au Sentier, la Galerie du Patrimoine restitue symboliquement la structure de l'intérieur d'une montre. (c) Manufacture Jaeger-LeCoultre

d'entreprise comme le lieu où l'entreprise se prend elle-même comme objet d'étude, s'exposant à des publics internes et externes (Meysonnat-Courtois, 1994). Le concept de «musée d'entreprise » est antinomique, car un musée est habituellement voué à la conservation des objets du passé, alors que l'entreprise en fabrique et en vend. Le musée d'entreprise peut toutefois être considéré comme une industrie culturelle proposant des services périphériques, développant des stratégies communicationnelles, à l'intention de publics identifiés, en concurrence ou complémentarité avec d'autres institutions culturelles.

Rares sont d'ailleurs les entreprises qui recourent au terme de «musée », synonyme pour beaucoup de passé et de poussière : on entend plus souvent parler de «Galerie du patrimoine », de « Maison » ou de termes équivalents. Les musées d'entreprise se situent à la jonction entre le domaine marchand, lucratif et mouvant, et celui plus traditionnel et culturel des musées publics. Lehman et Byrom (2007) les définissent comme des musées gérant des collections qui dépendent d'entreprises lucratives.

Un musée d'entreprise permet également à la société de se démarquer de la concurrence en justifiant que son image de marque est liée à son passé et à son enracinement dans un terroir (Rasse, 1995), ce qui crédibilise son ancrage dans la tradition.

Il est possible de dresser une typologie des lieux culturels créés par les entreprises, englobant notamment la notion de musées d'entreprise (MeysonnatCourtois, 1994) : les espaces de communication et les showrooms permettant de créer des événements 
valorisants en recourant à des expositions temporaires ou des événements clients ; les fondations constituées pour préserver un site ou encourager des créateurs ; les fondations qui «stockent » la mémoire des entreprises, sans forcément la rendre accessible au public ; les musées-galeries qui consistent en une collection permanente et en un espace de communication accessible au grand public, ou à des publics sélectionnés comme les écoles et les clients ; finalement, la catégorie la plus aboutie des musées d'entreprise qui couvrent une grande diversité de tailles, de natures des collections, de statuts, de domaines d'activité et de types d'organisation. Le secteur agroalimentaire est majoritairement représenté dans cette catégorie. Et, parmi les musées d'entreprise les plus connus, on trouve le domaine du luxe, comme les parfums ou les montres.

Plusieurs cas de figure peuvent être rencontrés pour les concepteurs de musées d'entreprise (MeysonnatCourtois, 1994). Si, en premier lieu, aucune contradiction n'est perçue entre l'entreprise et le musée, l'exposition est mise en place en se posant uniquement des questions d'ordre muséographique. En deuxième lieu, l'entreprise « tue » le musée, ce qui est le cas fréquent d'une collection détournée à des fins publicitaires lorsque les considérations commerciales prédominent celles de conservation du patrimoine, par exemple pour les entreprises horlogères qui mettent sur pied des expositions itinérantes lors des Grands Prix de Formule 1 ou des Jeux Olympiques. En troisième lieu, le musée « tue » l'entreprise, cas extrême, comme celui de la collection automobile des frères Schlumpf, dont la passion leur a fait mettre plus d'efforts et de ressources dans leur musée que dans leur entreprise, tombée ensuite en faillite. En dernier lieu, le musée d'entreprise a une existence réelle, une valeur intrinsèque comme l'Alimentarium de Vevey (Nestlé) ou le musée horloger de la manufacture Patek Philippe, à Genève.

Rigby (2008) mentionne différentes raisons pour lesquelles une société souhaite développer son propre musée : démontrer qu'elle fait partie de l'histoire, qu'elle est pionnière dans le développement de son industrie ; raconter une «belle histoire »; valoriser une marque « bien-aimée »; l'utiliser comme un outil de promotion des ventes. Les entreprises américaines, ouvrant des musées gratuits pour le public, sont plus friandes de ce concept que les entreprises européennes qui ont plutôt tendance à faire payer une entrée.
Les musées d'entreprise ne présentent pas exclusivement des objets liés à l'entreprise en elle-même, mais exposent des collections liées au produit, des aspects de la mémoire de la firme et des éléments relatifs plus largement au secteur industriel auquel elle appartient (Lehman et Byrom, 2007). Ils ont généralement pour vocation d'être une véritable destination touristique comme BMW ou Mercedes-Benz - mettant en œuvre technologie interactive et divertissement - comme Coca-Cola - pour promouvoir leur marque (McKay, 2007).

On distingue les musées d'entreprise à caractère technologique qui s'apparentent à des centres de culture scientifique, technique et industrielle, des musées d'entreprise à dimension entrepreneuriale, c'est-à-dire centrés sur l'enseigne industrielle, l'histoire de l'entreprise, le parcours économique, les produits et services (De La Broise, 1998). On trouve, dans le premier cas, le musée national des Télécommunications et Électropolis et, dans le second l'Alimentarium à Vevey dans lequel on met en exergue le continuum cohérent et riche d'informations entre l'entreprise - sa production, son appareil, ses marchés - et ses champs scientifiques. Ainsi, les différentes parties de l'exposition renvoient également à la biologie, la physiologie et la nutrition, la technique, la sociologie, l'histoire des techniques et l'ethnologie.

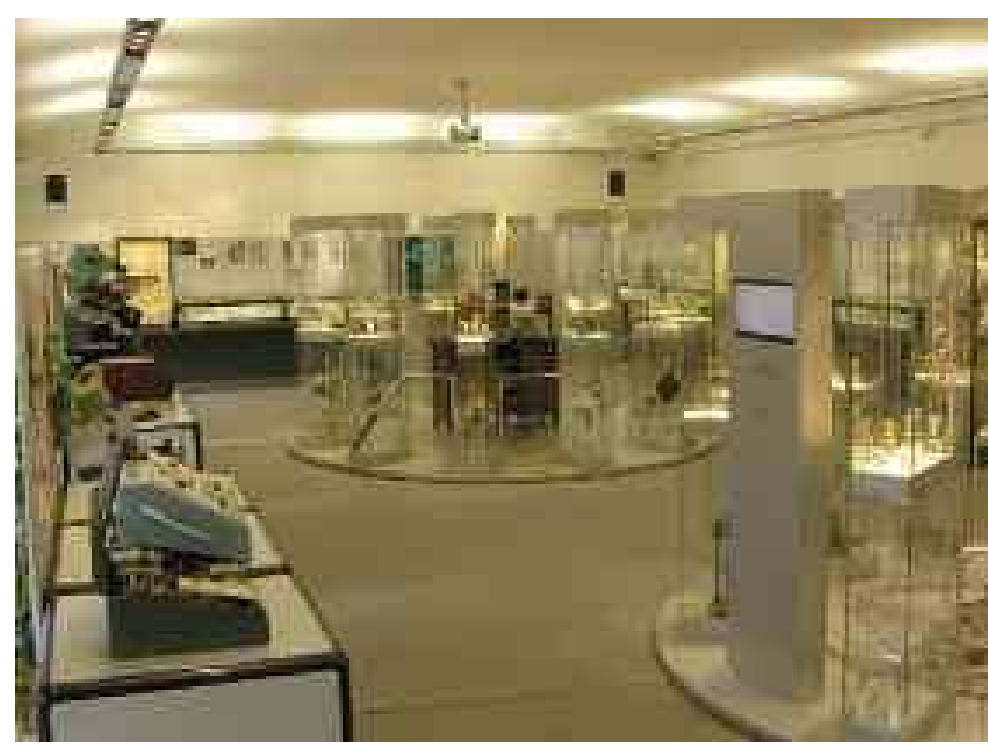

Outre sa collection de montres, le musée Omega renferme des appareils de chronométrage sportif, étant donné la longue collaboration de la marque avec le Comité International Olympique. 


\section{La visite d'entreprise}

La visite d'entreprise présuppose une ouverture à un ou plusieurs types de publics, mais elle n'implique pas nécessairement l'existence d'un musée. Par son ancienneté, son site, ses bâtiments, son équipement ou son savoir-faire ancestral, l'entreprise peut elle-même représenter une forme vivante d'écomusée, comme les entreprises viticoles opérant dans de vieux châteaux ou manoirs. Deux principales formes d'accueil et d'exhibitions au public sont à relever : la visite de site en activité qui prend un caractère de découverte nécessitant « de montrer du vrai » (De La Broise, 1998) et la journée portes ouvertes qui revêt un caractère événementiel, présentant l'entreprise dans son secteur d'activité et la rapprochant de son environnement immédiat. La visite d'entreprise peut être ponctuelle, périodique, commémorative ou inaugurale, voire de crise.

Morice (2006) et Pierre (2005) dressent, pour une entreprise, la liste des motivations pour l'ouverture de ses portes au public : outil de communication et de marketing au travers de la présentation d'un savoirfaire ; possibilité de développement d'une démarche commerciale pour augmenter ses ventes, surtout s'il existe un magasin de vente de produits à l'issue de la visite ; occasion de valoriser le travail des employés et d'assurer la promotion des métiers auprès des jeunes ou des demandeurs d'emploi ; opportunité pour favoriser la cohésion sociale, en créant un lien entre les employés ; accès au grand public, d'une manière exceptionnelle et sur une période déterminée, au cœur de l'entreprise ; finalement, la visite d'entreprise valorise les forces et les traditions économiques et sociales d'une région, d'une manière dynamique et non passéiste.

Les visites d'entreprise permettent de construire une expérience de visite, fondée sur la communication d'un contenu étoffé, tonique, stimulant et suscitant la réflexion (Rivard, 2006). Les secteurs de l'agroalimentaire, de l'artisanat et des entreprises liées à la thématique environnementale constituent des filières captives de la visite d'entreprise en raison des enjeux de commercialisation et de communication (Pierre, 2005). La visite d'entreprise peut véritablement constituer un produit touristique, intégré par exemple dans un circuit de découverte des produits régionaux en insérant des visites d'entreprises significatives d'un terroir qui répond véritablement à une attente des visiteurs, comme les distilleries de la Speyside Valley, en Écosse. De plus, cette filière s'inscrit dans une approche alternative et participative du tourisme :

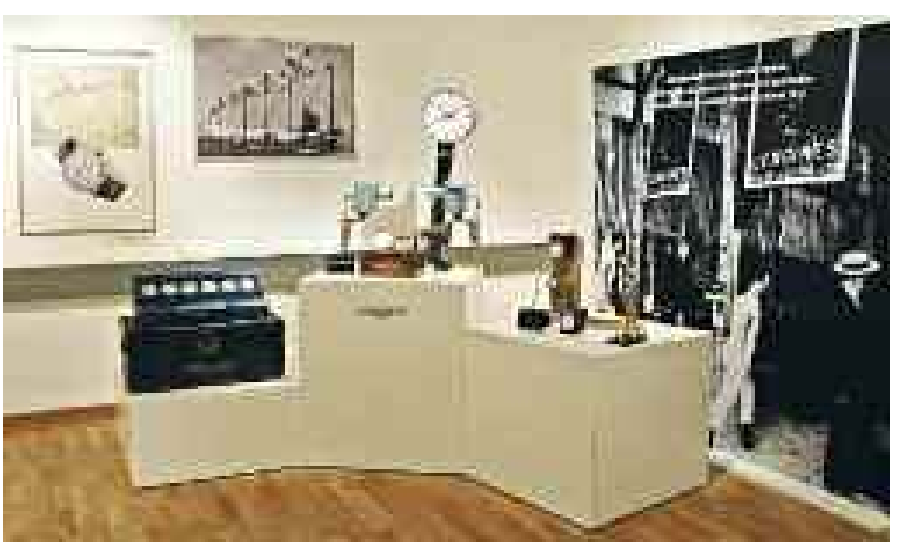

Le musée Longines, à Saint-Imier, plonge le visiteur dans l'univers du chronométrage sportif. (c) Compagnie des Montres Longines Francillon SA

découvrir une région de l'intérieur, être en relation avec ses habitants, mettre au premier plan les composantes de l'identité des territoires.

Au travers de la visite d'entreprise ou du musée d'entreprise, Ksouri, Marcon \& Prieulh (2001) structurent l'offre de découverte économique en trois composantes : les services de base, indispensables pour répondre à la demande générique des visiteurs (service d'accueil, visites guidées, supports à la visite, scénographie) ; en deuxième lieu, les services annexes, qui étoffent et valorisent la visite (magasin de vente, musées ou espaces culturels, salle de projection); finalement, les services périphériques, qui complètent l'offre (prestations touristiques comme le transport, l'hébergement, la restauration). Pour la Suisse, Ksouri et al. (2000) mettent en évidence la volonté de miser sur son savoir-faire en matière d'horlogerie. Un dépliant « La Route de l'Horlogerie » met d'ailleurs en valeur 22 musées et manufactures situés dans l'Arc jurassien, de Genève à Schaffhouse.

\section{Des musées d'entreprise aux musées $d$ 'entreprises horlogères}

En France comme en Suisse, le paysage des musées d'entreprise s'avère extrêmement hétérogène, correspondant à la diversité des sociétés fondatrices (Meysonnat-Courtois, 1994). Notre propos n'est pas de recenser et de classer les musées d'entreprise, mais de comprendre pourquoi on assiste à une valorisation croissante de ce patrimoine industriel et culturel.

De nombreuses entreprises disposent déjà de collections d'objets et de documents, plus ou moins bien mis en valeur. De plus en plus de manufactures horlogères créent des espaces consacrés à leur patrimoine, de la 


\begin{tabular}{|c|c|c|c|c|c|}
\hline $\begin{array}{l}\text { Ouverture } \\
\text { du musée }\end{array}$ & 1849 & 1991 & 2008 & 1983 & 1992 \\
\hline $\begin{array}{l}\text { Raison de la } \\
\text { création } \\
\text { d'un musée }\end{array}$ & $\begin{array}{l}\text { Conservation du } \\
\text { patrimoine } \\
\text { et instrument } \\
\text { pédagogique }\end{array}$ & $\begin{array}{l}\text { Commémoration } \\
\text { des } 200 \text { ans de la } \\
\text { manufacture }\end{array}$ & $\begin{array}{l}\text { Faire connaître } \\
\text { le rôle de pionnier } \\
\text { de l'entreprise }\end{array}$ & $\begin{array}{l}\text { Mise en valeur du } \\
\text { patrimoine, } \\
\text { légitimation de la } \\
\text { marque, outil de } \\
\text { relations publiques }\end{array}$ & $\begin{array}{l}\text { Positionner la } \\
\text { marque sur son } \\
\text { segment, légitimer } \\
\text { le patrimoine et } \\
\text { l'héritage de la marque } \\
\text { dans le groupe Swatch }\end{array}$ \\
\hline Publics-cibles & $\begin{array}{l}\text { Grand public: } \\
\text { surtout scolaires, } \\
\text { passionnés, personnes } \\
\text { âgées, touristes }\end{array}$ & $\begin{array}{l}\text { Public restreint : } \\
\text { clients, presse, } \\
\text { étudiants } \\
\text { (futurs employés) }\end{array}$ & $\begin{array}{l}\text { Public restreint : } \\
\text { personnel, clients, } \\
\text { journalistes, VIP }\end{array}$ & $\begin{array}{l}\text { Grand public (sur } \\
\text { réservation), clients, } \\
\text { presse, } \\
\text { collectionneurs }\end{array}$ & $\begin{array}{l}\text { Visites adaptées } \\
\text { aux niveaux et aux } \\
\text { attentes, du VIP } \\
\text { aux écoles }\end{array}$ \\
\hline $\begin{array}{l}\text { Visites } \\
\text { d'entreprise }\end{array}$ & (ne s'applique pas) & $\begin{array}{l}\text { Complémentarité } \\
\text { avec le musée }\end{array}$ & $\begin{array}{l}\text { Visite des ateliers } \\
\text { réservée aux VIP } \\
\text { complément du musée }\end{array}$ & $\begin{array}{l}\text { Non (quelques } \\
\text { exceptions) }\end{array}$ & $\begin{array}{l}\text { Pour détaillants, } \\
\text { journalistes, en } \\
\text { du Patrimoine }\end{array}$ \\
\hline $\begin{array}{l}\text { Rôle du musée } \\
\text { dans la } \\
\text { communication } \\
\text { de l'entreprise }\end{array}$ & $\begin{array}{l}\text { Instrument de } \\
\text { promotion aussi pour } \\
\text { une ville et une } \\
\text { région }\end{array}$ & $\begin{array}{l}\text { Complète la visite } \\
\text { d'entreprise, pour } \\
\text { montrer que cette } \\
\text { dernière travaille dans } \\
\text { une tradition innovante }\end{array}$ & $\begin{array}{l}\text { Légitime et intègre } \\
\text { le patrimoine dans } \\
\text { la communication } \\
\text { de l'entreprise }\end{array}$ & $\begin{array}{l}\text { Permet des opérations } \\
\text { de relations publiques } \\
\text { internes (personnel, } \\
\text { détaillants) et externes } \\
\text { (VIP, presse, autorités) }\end{array}$ & $\begin{array}{l}\text { Actuellement, de manière } \\
\text { informelle. Va s'ancrer à } \\
\text { l'avenir dans le } \\
\text { patrimoine immatériel } \\
\text { de la marque }\end{array}$ \\
\hline $\begin{array}{l}\text { Contradiction } \\
\text { entre } \\
\text { conservation } \\
\text { et marketing }\end{array}$ & $\begin{array}{l}\text { Non, le marketing } \\
\text { n'est pas l'ennemi } \\
\text { des historiens }\end{array}$ & $\begin{array}{l}\text { Non, le passé est } \\
\text { source d'inspiration } \\
\text { pour l'innovation }\end{array}$ & $\begin{array}{l}\text { Éventuellement : } \\
\text { l'histoire peut faire } \\
\text { vendre, le marketing } \\
\text { peut réorienter l'histoire }\end{array}$ & $\begin{array}{l}\text { Non, le musée } \\
\text { apporte de la } \\
\text { légitimité }\end{array}$ & $\begin{array}{l}\text { Non, car le } \\
\text { positionnement est } \\
\text { basé sur l'élégance } \\
\text { et la tradition }\end{array}$ \\
\hline $\begin{array}{l}\text { Place de } \\
\text { la scénographie }\end{array}$ & $\begin{array}{l}\text { Plusieurs scénogra- } \\
\text { phies simultanées, } \\
\text { projets interactifs } \\
\text { (i-touch, films 3D) }\end{array}$ & $\begin{array}{l}\text { Concept } \\
\text { chronologique, } \\
\text { avec interactivité, } \\
\text { pour suppléer au } \\
\text { guide }\end{array}$ & $\begin{array}{l}\text { Immersion dans } \\
\text { l'identité de la mar- } \\
\text { que avec matériaux } \\
\text { choisis. Films sur } \\
\text { écran circulaire }\end{array}$ & $\begin{array}{l}\text { Scénographie } \\
\text { thématique } \\
\text { et classique, pièces en } \\
\text { vitrines avec cartons }\end{array}$ & $\begin{array}{l}\text { Plusieurs espaces } \\
\text { thématiques, scénographie } \\
\text { classique, montres } \\
\text { contextualisées } \\
\text { en vitrines }\end{array}$ \\
\hline Portes ouvertes & (ne s'applique pas) & $\begin{array}{l}\text { Oui, lors des journées } \\
\text { du patrimoine horloger }\end{array}$ & $\begin{array}{l}\text { Oui, lors des journées } \\
\text { du patrimoine horloger }\end{array}$ & $\begin{array}{l}\text { Non } \\
\text { un jubilé }\end{array}$ & $\begin{array}{l}\text { Oui, pour commémorer } \\
\text { du personnel }\end{array}$ \\
\hline $\begin{array}{l}\text { Tendance à } \\
\text { l'apparition de } \\
\text { nouveaux musées } \\
\text { d'entreprises } \\
\text { horlogères }\end{array}$ & $\begin{array}{l}C^{\prime} \text { est d'abord une } \\
\text { mode, maintenant } \\
\text { tendance de fond }\end{array}$ & $\begin{array}{l}\text { Les musées deviennent } \\
\text { un outil de promotion } \\
\text { des ventes }\end{array}$ & $\begin{array}{l}\text { Conséquence d'une } \\
\text { course dans le luxe } \\
\text { Permet du relationnel } \\
\text { avec les collectioneurs } \\
\text { et les vendeurs. Donne } \\
\text { un élan de recherche }\end{array}$ & $\begin{array}{l}\text { Ce développement } \\
\text { n'est pas choquant, } \\
\text { il met en avant une } \\
\text { longue tradition }\end{array}$ & $\begin{array}{l}\text { Engouement pour la montre } \\
\text { de la part du public. } \\
\text { Les musées privés assoient } \\
\text { une légitimité et une } \\
\text { crédibilité à la marque }\end{array}$ \\
\hline $\begin{array}{l}\text { Spécificité du } \\
\text { musée concerné }\end{array}$ & $\begin{array}{l}\text { Collections condensées } \\
\text { et thématiques, } \\
\text { accessibles au profane }\end{array}$ & $\begin{array}{l}\text { Aspect didactique } \\
\text { grâce à la visite de la } \\
\text { manufacture qui com- } \\
\text { plète celle du musée }\end{array}$ & $\begin{array}{l}\text { Design futuriste, } \\
\text { rappelant l'identité } \\
\text { et la vie de la } \\
\text { marque. }\end{array}$ & $\begin{array}{l}\text { Premier musée } \\
d^{\prime} \text { entreprise } \\
\text { horlogère du monde. }\end{array}$ & $\begin{array}{l}\text { Mise en valeur d'un } \\
\text { patrimoine dont le } \\
\text { contenu peut être traité par } \\
\text { une approche scientifique }\end{array}$ \\
\hline
\end{tabular}




\begin{tabular}{|c|c|c|c|c|}
\hline $\begin{array}{l}\text { Jaeger-LeCoultre } \\
\text { (privé, groupe Richemont) }\end{array}$ & $\begin{array}{l}\text { AudemarsPiguet } \\
\text { (privé, indépendant) }\end{array}$ & $\begin{array}{l}\text { Museum } \\
\text { Développement } \\
\text { (cabinet privé) }\end{array}$ & $\begin{array}{l}\text { Chopard } \\
\text { (privé, indépendant) }\end{array}$ & $\begin{array}{l}\text { Vacheron Constantin } \\
\text { (privé, groupe Richemont) }\end{array}$ \\
\hline 2007 & 1990 & (ne s'applique pas) & $\begin{array}{l}2006 \text { : ouverture } \\
\text { du L.U.Ceum (muséum } \\
\text { Louis Ulysse Chopard) }\end{array}$ & $\begin{array}{l}\text { Collection itinérante } \\
\text { Pièces aussi visibles à la } \\
\text { Maison Vacheron, Genève }\end{array}$ \\
\hline $\begin{array}{l}\text { La Galerie du } \\
\text { Patrimoine s'inscrit } \\
\text { dans un parcours } \\
\text { de découverte de } \\
\text { la marque }\end{array}$ & $\begin{array}{l}\text { Prouver le grand } \\
\text { passé de } \\
\text { l'horlogerie à } \\
\text { la Vallée de Joux }\end{array}$ & $\begin{array}{l}\text { Demande de } \\
\text { la part de clients }\end{array}$ & $\begin{array}{l}\text { Réflexion sur le temps } \\
\text { et mise en valeur de } \\
\text { la collection privée } \\
\text { de K.-F. Scheufele }\end{array}$ & $\begin{array}{l}\text { Valorisation de pièces } \\
\text { plus que centenaires et } \\
\text { nouvelles acquisitions }\end{array}$ \\
\hline $\begin{array}{l}\text { Complémentarité } \\
\text { avec la Galerie }\end{array}$ & $\begin{array}{l}\text { Complémentarité } \\
\text { avec le musée }\end{array}$ & (ne s'applique pas) & $\begin{array}{l}\text { Limitées et précédant } \\
\text { souvent la visite du musée }\end{array}$ & $\begin{array}{l}\text { Sur les sites de Plan-les-Ouates } \\
\text { et de Joux.Ouverture sur } \\
\text { demande de groupes }\end{array}$ \\
\hline $\begin{array}{l}\text { Outil de marketing } \\
\text { précieux et complet : } \\
\text { la communication } \\
\text { publicitaire puise dans } \\
\text { I'histoire et le patrimoine }\end{array}$ & $\begin{array}{l}\text { Il fait partie } \\
\text { intégrante du } \\
\text { concept marketing: } \\
\text { teaser à la vente }\end{array}$ & $\begin{array}{l}\text { Il peut constituer } \\
\text { un nouveau canal } \\
\text { de valorisation } \\
\text { commerciale, voire } \\
\text { de vente }\end{array}$ & $\begin{array}{l}\text { Espace de communication } \\
\text { pour les clients, affirmation } \\
\text { des valeurs de l'entreprise. } \\
\text { Également outil de } \\
\text { communication interne }\end{array}$ & $\begin{array}{l}\text { Outil de communication } \\
\text { de la pérennité de Vacheron, } \\
\text { fonctionnant depuis } 1755 \\
\text { sans interruption. L'histoire } \\
\text { est source de crédibilité }\end{array}$ \\
\hline $\begin{array}{l}\text { Véhicule les valeurs de } \\
\text { l'entreprise. Comme } \\
\text { pour une publicité, } \\
\text { un brief a été établi }\end{array}$ & $\begin{array}{l}\text { Scénographie } \\
\text { thématique et } \\
\text { chronologique } \\
\text { avec participation } \\
\text { d'un guide }\end{array}$ & $\begin{array}{l}\text { Les musées privés } \\
\text { ont plus de moyens } \\
\text { pour des scéno- } \\
\text { graphies originales } \\
\text { et interactives }\end{array}$ & $\begin{array}{l}\text { Concept basé sur le } \\
\text { nombre d'or } 12 . \\
\text { Intégration de groupes } \\
\text { de vitrines dans la } \\
\text { charpente du toit }\end{array}$ & $\begin{array}{l}\text { Tout le matériel d'exposition } \\
\text { est « prémâché » pour les } \\
\text { filiales : vitrines, place } \\
\text { des pièces, textes... }\end{array}$ \\
\hline Oui, pour familles & Rarement & (ne s'applique pas) & $\begin{array}{l}\text { Non, pour préserver } \\
\text { l'exclusivité et la sécurité }\end{array}$ & $\begin{array}{l}\text { Toute personne peut voir } \\
\text { la collection chez les filiales }\end{array}$ \\
\hline $\begin{array}{l}\text { Les musées publics, } \\
\text { communiquent l'histoire } \\
\text { horlogère ; les privés } \\
\text { valorisent le patrimoine } \\
d^{\prime} \text { 'une marque, selon une } \\
\text { logique de communication }\end{array}$ & $\begin{array}{l}\text { Le musée sert } \\
\text { à légitimer l'existence } \\
\text { et l'histoire de la } \\
\text { marque. Le terme } \\
\text { showroom pourrait } \\
\text { être plus adapté }\end{array}$ & $\begin{array}{l}\text { La tendance vient } \\
\text { d'Amérique du Nord. } \\
\text { Pas de différence de } \\
\text { conception avec les } \\
\text { autres types de musées }\end{array}$ & $\begin{array}{l}\text { Perçue positivement et } \\
\text { permettant des échanges } \\
\text { et des collaborations } \\
\text { avec les musées publics }\end{array}$ & $\begin{array}{l}\text { Pour beaucoup d'entreprises, } \\
c^{\prime} \text { est un alibi marketing }\end{array}$ \\
\hline $\begin{array}{l}\text { Les pièces présentées ont } \\
\text { toutes été conçues et } \\
\text { fabriquées par } \\
\text { la Manufacture }\end{array}$ & $\begin{array}{l}\text { Lien au terroir, en } \\
\text { retraçant l'histoire } \\
\text { de la marque et de } \\
\text { la Vallée de Joux }\end{array}$ & (ne s'applique pas) & $\begin{array}{l}\text { Collection privée, avec } \\
d^{\prime} \text { autres pièces que } \\
\text { des Chopard }\end{array}$ & $\begin{array}{l}\text { Pas de collection réunie } \\
\text { dans un seul lieu }\end{array}$ \\
\hline
\end{tabular}




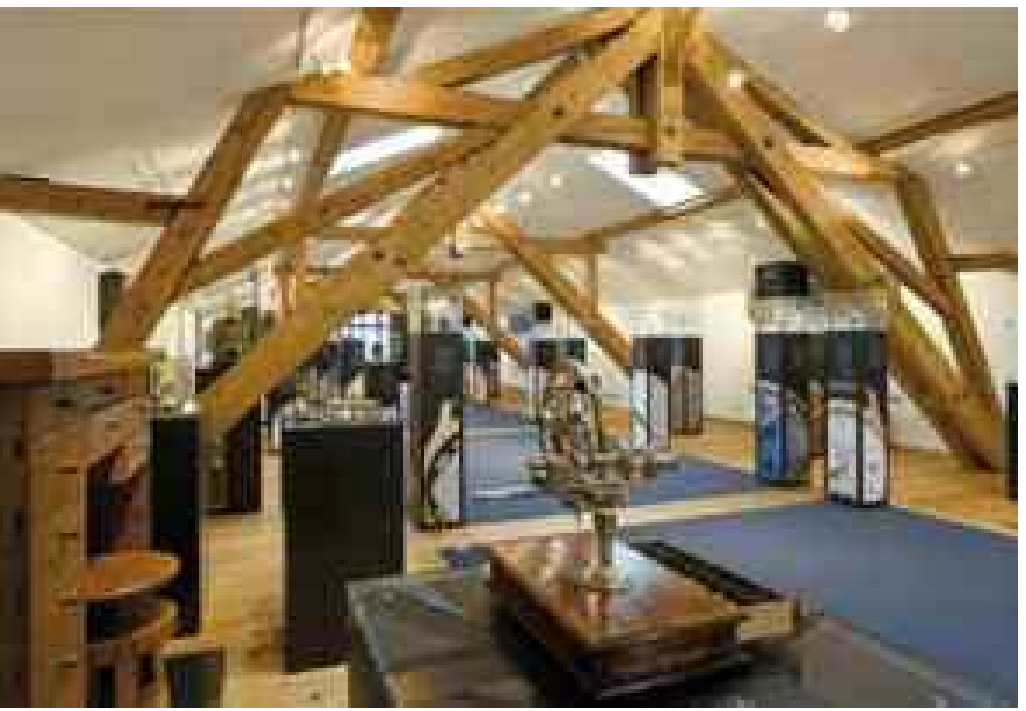

En douze espaces thématiques, le L.U.Ceum, à Fleurier, présente la collection personnelle de Karl-Friedrich Scheufele, CEO de Chopard. (c) François Bertin, Grandvaux, Suisse

- le musée Girard-Perregaux, situé à la villa Marguerite, à La Chaux-de-Fonds, a été fermé en 2007 suite au vol de plusieurs pièces, heureusement retrouvées. Après sécurisation et transformation, il rouvrira fin 2009. De plus, le conservateur Willy Schweizer, est responsable d'un deuxième bâtiment, le musée Jean Richard, consacré aux machines et à l'outillage horloger, situé dans la villa Schwob; ce dernier met en valeur le savoir-faire depuis le paysan-horloger ou « rlodgeur » (Jelmini, 2007), jusqu’à l'actuel ingénieur-designer ;

- le musée TAG Heuer $\mathbf{3 6 0}$ est le plus récent et le plus high tech des musées étudiés en termes de scénographie pour plonger le visiteur dans l'univers de la marque. La scénographie, s'appuyant sur des matériaux très design rappelant les codes génétiques de la marque, ainsi que sur des projections sur écran circulaire présentant les « héros de l'entreprise », est très «immersive ». Sa conservatrice, Mathilde Tournois, porte le titre de brand heritage manager, ce qui montre bien son ancrage dans la stratégie marketing de l'entreprise ;

- à l'opposé de TAG Heuer, le musée Omega est celui qui est ouvert depuis le plus longtemps (1983). Cela se remarque par sa scénographie où la quantité de pièces exposées (près de 2000 ) nuit quelque peu à la vision d'ensemble d'une marque très active depuis longtemps dans de nombreux secteurs, notamment le chronométrage sportif, la conquête de l'espace et l'aventure lunaire. Le conservateur, Marco Richon, fait également des recherches sur la marque et a publié de nombreux ouvrages et documents de référence. Le musée fournit aussi de la documentation aux personnes qui la demandent. En cela, le musée Omega est celui qui se rapproche le plus des quatre missions de l'ICOM évoquées plus haut. C'est aussi celui qui est le plus largement ouvert au grand public. Les mêmes considérations sur la recherche et la documentation s'appliquent au musée Longines (faisant également partie du groupe Swatch), grâce aux publications de son conservateur, Patrick Linder ;

- le musée Jaeger-LeCoultre s'appelle « la Galerie du Patrimoine » : il a pour particularité de se situer dans le bâtiment où le fondateur Antoine LeCoultre avait son atelier. Comme pour le musée TAG Heuer, avec les projecteurs vidéo en moins, la scénographie de la Galerie du Patrimoine est immersive et donne l'impression d'entrer dans le mouvement d'une montre ;

- le musée Audemars Piguet est également installé dans une demeure historique de la famille Audemars adjacente à la manufacture. De plus, il reflète l'histoire de la Vallée de Joux ;

- le musée Chopard (le L.U.Ceum, jeu de mots avec lyceum et les initiales de Louis-Ulysse Chopard) a comme spécificité d'être avant tout le lieu d'exposition de la collection personnelle de Karl-Friedrich Scheufele, co-propriétaire de l'entreprise. La collection Chopard comporte de nombreuses pièces qui n'ont pas été produites par la manufacture, mais donnent l'opportunité d'une réflexion technique et philosophique sur le temps ;

- la collection Vacheron Constantin est atypique dans notre échantillon : la marque dispose d'une Maison Vacheron, sise au cœur historique de Genève, c'est un point de vente avec quelques pièces historiques exposées, mais elle ne désire pas figer ses collections dans un musée. Par contre, elle a une collection qui tourne dans ses filiales, avec des vitrines adéquates, pour apporter l'esprit et les valeurs de la marque aux clients de ses différents marchés ;

- une collaboratrice de Museum Development a été interrogée dans le but d'avoir l'avis externe d'une société de conception muséale pour créer un discours avec la collaboration d'une équipe pluridisciplinaire. Johanne Blanchet, Québécoise, a également l'expérience des musées et centres d'interprétation d'Amérique du Nord.

\section{Conclusion et perspectives}

Faisons maintenant le point sur notre question de recherche : il ressort de notre analyse que toutes les personnes interrogées voient les musées d'entreprises horlogères remplir naturellement une double fonction : la conservation d'un patrimoine collectif et la promotion d'une marque, voire du savoir-faire d'une 
région (la vallée de Joux, les Montagnes neuchâteloises, la région de Bienne-Seeland). Aucun conservateur ne perçoit de contradiction gênante entre l'histoire et le marketing, si ce n'est que ce dernier pourrait, dans certains cas, être soupçonné « d'arranger l'histoire » à des fins commerciales : par exemple, ne pas parler d'échecs commerciaux ou de périodes plus sombres de la vie des entreprises, comme pendant les années de guerre.

Les éléments invariants entre les répondants sont largement majoritaires, comme les raisons qui ont présidé à l'ouverture d'un musée ou à la présentation de collections, le rôle du musée pour asseoir la communication de l'entreprise dans une temporalité historique, ou encore le lien sélectif des publics habilités à faire une visite d'entreprise : peu de portes sont d'ailleurs ouvertes hors des Journées du patrimoine horloger.

Les éléments variants portent surtout sur les publicscibles, plus larges pour certains musées et collections, comme Omega, Longines ou Vacheron Constantin, sur la scénographie qui va du très traditionnel (Omega, Longines) au très immersif (TAG Heuer, Jaeger-LeCoultre) et sur la mise en valeur de la région, plus évidente pour le musée public que pour les musées privés. Finalement, la spécificité de chaque musée est le principal élément variant, constituant pour chacun un axe de positionnement. Par exemple: Omega peut se targuer d'être le premier musée d'entreprise horlogère au monde ; Audemars Piguet a un musée qui fait le lien avec le terroir régional ; Vacheron Constantin dispose de collections exclusivement itinérantes.

Nous émettons l'hypothèse que, parmi les facteurs sous-jacents à ces opinions concordantes, il y avait d'une part la demande d'inscription de l'urbanisme horloger des Montagnes neuchâteloises au patrimoine mondial de l'UNESCO (ratifiée en juin 2009) qui a eu un effet accélérateur de valorisation du Patrimoine, et d'autre part la volonté des manufactures horlogères d'immerger le plus intensément possible leurs clients dans l'univers de leur marque. Finalement, pour les musées publics, comme le musée d'horlogerie du Locle, il s'agit de développer une véritable réflexion de marketing territorial autour du thème de « la Culture horlogère ». Du côté de la scénographie, on observe de grandes différences qui vont de l'alignement de montres munies de cartons explicatifs dans de classiques vitrines (Omega, Longines) à des mises en valeur très high tech avec installations multimédias (TAG Heuer) et matériaux de haut de

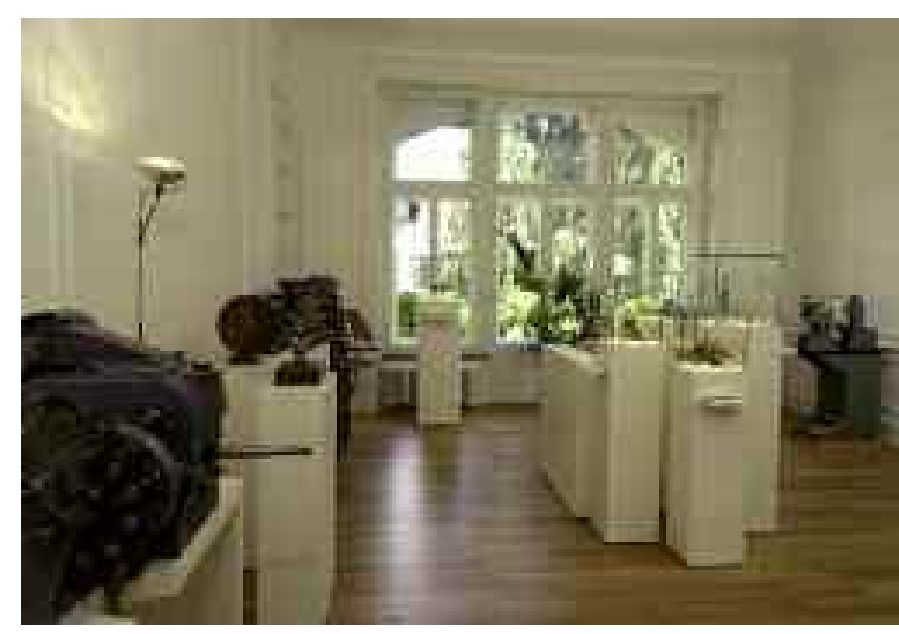

Outre un musée de montres, la manufacture Girard-Perregaux, à La Chaux-de-Fonds dispose à la Villa Jean Richard $d^{\prime}$ un musée de machines et d'outillage horloger unique au monde. (C) Jean Richard

gamme (Audemars Piguet, Jaeger-LeCoultre, GirardPerregaux). Les choix scénographiques nous semblent être autant une question de moyens financiers alloués que le reflet de l'époque à laquelle les expositions ont été conçues.

Une recherche sur Internet nous indique que tous les musées étudiés sont mentionnés ou accessibles sur le site des entreprises correspondantes, de manière plus ou moins développée : Omega, Vacheron Constantin (une page fixe), musée d'Horlogerie du Locle (plusieurs pages fixes), Girard-Perregaux, Jaeger-LeCoultre et Chopard (images défilantes), Audemars Piguet (accès à une visite guidée, en ligne, par son conservateur Martin Wehrli), TAG Heuer et Longines (visite virtuelle complète). Clairement, pour ces deux dernières marques, la volonté managériale affichée est de plonger les visiteurs qui n'ont pas forcément accès au musée réel dans les racines de la marque. Il faut relever ici que les musées d'entreprise étudiés ne perçoivent pas de droit d'entrée : la presse, les distributeurs et les collectionneurs y sont naturellement invités gracieusement, de même que des groupes d'intérêt, par exemple les écoles professionnelles, sur rendez-vous. Seul le musée Patek Philippe, à Genève, ouvert très largement au grand public comme le musée international d'Horlogerie de la Chaux-deFonds celui du Locle - tous les deux sous gestion publique - pratiquent un tarif d'entrée de 10 francs pour les adultes, soit environ 6,50 euros. La gratuité d'entrée des musées étudiés fait donc partie de la stratégie de promotion de la marque, le visiteur étant considéré comme un partenaire, voire un ami. 
Cette recherche exploratoire nous apparaît riche de perspectives futures, notamment en étendant nos entretiens à d'autres musées comme le Patek Philippe Museum, la Cité du Temps (groupe Swatch) ou encore le musée IWC, mais aussi en interrogeant des visiteurs (détaillants, clients, VIP ou grand public) de musées privés et publics sur leurs perceptions par rapport aux marques, à leurs valeurs et à leur patrimoine. En outre, un benchmarking des différents musées d'entreprises horlogères pourrait déboucher sur l'identification de bonnes pratiques destinées à aider des entreprises qui n'ont pas encore de musée, mais qui réfléchissent à en ouvrir un comme élément de leur stratégie de communication, à l'instar de Tissot, au Locle.

Nous avons également identifié dans ces musées un certain nombre de scénographies innovantes, plus ou moins interactives, comme chez TAG Heuer et Jaeger-LeCoultre, qui méritent d'être explorées du point de vue de leur perception par les visiteurs. Finalement, les offices du tourisme et syndicats d'initiative pourraient être intéressés, dans une démarche de marketing horloger de l'Arc jurassien, à réfléchir au concept supra régional - voire supra national, en incluant la Franche-Comté - d'une véritable « Route de l'Horlogerie » mettant en réseau, à la manière des « Routes du Vin », les musées d'entreprises horlogères s'ouvrant au public hors des journées du patrimoine présentées plus haut.

Les auteurs tiennent à remercier les personnes interrogées lors de cette étude: Cécile Aguillaume, conservatrice (jusqu'en juillet 2009) du musée d'Horlogerie du Locle (www.mhl-monts.ch); Dominique Bernaz, directeur de la Maison Vacheron Constantin à Genève (www.vacheronconstantin.com) ; Johanne Blanchet, chef de projet à Museum Development/Thématis (cabinet de conseil en muséographie) à Neuchâtel et Montreux (www.museum.ch); Patrick Linder, historien consultant chez Longines à Saint-Imier (www.longines.com); Marco Richon, conservateur du musée Omega à Bienne (www.omega.ch); Willy Schweizer, conservateur du musée Girard-Perregaux à La Chaux-de-Fonds (www.girard-perregaux.com) ; Mathilde Tournois, Brand Heritage Manager à TAG Hener à La Chaux-de-Fonds (www.taghener.com); Sébastien Vivas, responsable patrimoine à JaegerLeCoultre, Le Sentier (www.jaeger-lecoultre.com); Anne Walther, archiviste et conservatrice chez Chopard à Fleurier (www.chopard.com) et Martin Wehrli, Director, Museum \& Heritage à Audemars Piguet, Le Brassus (www.audemarspiguet.com).

\section{Nołes}

(1) Statuts de l'ICOM, art. 2, alinéa b

(2) www.urbanismehorloger.ch

\section{Bibliographie}

Debraine, L. Musées horlogers : le temps privatisé, Le Temps, 18 octobre 2007.

Debraine, L. Musées publics ou musées privés ?, HH Journal, édition 12, 22 janvier 2008.

De la Broise, P. Accueil culturel et mises en scène de l'entreprise. Thèse de doctorat, université de Bourgogne, 1998.

FHS : Fédération de l'industrie horlogère suisse : www.fhs.ch/fr/statistics.php (consulté le 12 août 2009).

Jelmini, J.-P. La Culture horlogère, in La Culture horlogère : art, industrie, marketing, coordonné par K. Zorik et F. Courvoisier, Neuchâtel : Ed. JIMH, 2007.

Ksouri, S., Marcon, A. et Preuilh P. Tourisme de découverte économique et visites d'entreprises. Paris : La Documentation française, 2001.

Lehman, K., et Byrom, J. Corporate museums in Japan: institutionalising a culture of industry and technology, Proceedings of the $9^{\text {th }}$ AIMAC Conference, university of Valencia, Spain, July 2007.

McKay, B. Coke leads trend of bigger, flashier corporate museums, The Wall Street Journal, May 21, 2007.

Meysonnat-Courtois, P. Les musées d'entreprise en France : une réalité à dépasser, un concept à inventer, la lettre de l'OCIM, n³4, 1994, pp. 13-17.

Morice, J.-R. La visite d'entreprise en Europe. Un champ à explorer, Cahier Espaces, n92, 2006, pp. 10-15.

Nardin, L. En quête de prestige, les horlogers créent leurs musées, Luxes par Bilan, $\mathrm{n}^{\circ} 13,2007$, pp. 23-26.

Pierre, C. Du tourisme industriel à la visite d'entreprises, la lettre de l'OCIM, n¹01, 2005, pp. 18-25.

Prothery, L. Dans le secret des musées horlogers, L’Express.fr, 15 avril 2008.

Rasse, P. Les musées d'entreprise, quelle médiation de l'histoire ?, Communication et organisation, $\mathrm{n}^{\circ}$, 1995, pp. 71-84.

Roche, D. La communication externe, enjeu majeur de l'ouverture des entreprises, Cahier Espaces, n²92, 2006, pp. 19-20.

Rigby, R. Companies make an exhibition of themselves. Names from Spam to Zippo like to showcase their history, Financial Time, February 26, 2008

Rivard, R. Construire une expérience de visite. Ressentir, apprendre, s'interroger, Cahier Espaces, n92, 2006, pp. 114-116.

Tissier, P. Le patrimoine horloger des villes du Haut s'ouvre au public, Courrier Neuchâtelois, 17 octobre 2007, p. 2. 